\title{
Detection Issues with Many BS Antennas Available for Bandwidth-Efficient Uplink Transmission in a MU-MIMO System
}

\author{
${ }^{(1)}$ Paulo Torres, ${ }^{(2)}$ Antonio Gusmao \\ (1) IPCB - Instituto Politecnico de Castelo Branco, Portugal \\ ${ }^{(2)}$ IST - Instituto Superior Tecnico, Universidade de Lisboa, Portugal \\ ${ }^{(1)}$ paulo.torres@ipcb.pt, ${ }^{(2)}$ gus@ist.utl.pt
}

\begin{abstract}
This paper is concerned with Single Carrier (SC)/Frequency Domain Equalization (FDE) for bandwidthefficient uplink block transmission, with Quadrature Amplitude Modulation (QAM) schemes, in a Multi-User (MU) Multi-Input Multi-Output (MIMO) system. The number of Base Station (BS) receiver antennas is assumed to be large, but not necessarily much larger than the overall number of transmitter antennas jointly using the same time/frequency resource at Mobile Terminals (MT).

In this context, we consider several detection techniques and evaluate, in detail, the corresponding detection performances (discussed with the help of selected performance bounds), for a range of values regarding the number of available $B S$ receiver antennas. From our performance results, we conclude that simple linear detection techniques, designed to avoid the need of complex matrix inversions, can lead to unacceptably high error floor levels; however, by combining the use of such simple linear detectors with an appropriate interference cancellation procedure - within an iterative Decision-Feedback (DF) technique -, a close approximation to the Single-Input Multi-Output (SIMO) Matched-Filter Bound (MFB) performance can be achieved, even for 64-QAM schemes, after a few iterations.

Index Terms-Broadband MU-MIMO systems; massive MIMO; uplink; SC/FDE; linear detection; iterative DF detection; performance evaluation, bandwidth efficiency.
\end{abstract}

\section{INTRODUCTION}

Cyclic-Prefix (CP)-assisted block transmission schemes, proposed and developed for broadband wireless systems, take advantage of current low-cost, flexible, Fast Fourier Transform (FFT)-based signal processing technology, with both Orthogonal Frequency Division Multiplexing (OFDM) and SC/FDE [1]. Mixed air interface solutions (OFDM for downlink and SC/FDE for uplink [1]) are now widely accepted.

The development of MIMO technologies has been crucial for the "success story" of broadband wireless communications. Through spatial multiplexing, following and extending ideas early presented in [2], MIMO systems are currently able to provide very high bandwidth efficiencies and a reliable radio transmission at data rates beyond $1 \mathrm{Gigabit} / \mathrm{s}$. In the last decade, MU-MIMO systems have been successfully implemented and introduced in several broadband communication standards [3]; in such "space division mutiple access" systems, the more antennas the BS is equipped with, the more users can jointly communicate in the same time-frequency resource.
The adoption of MU-MIMO systems with a very large number of antennas in the BS, much larger than the number of MT antennas in its cell, was proposed in [4]. This "massive MIMO" approach was shown to be recommendable [4], [5], [6]: simple linear processing for MIMO detection/precoding (uplink/downlink), becomes quasi-optimal; both Multi-User Interference (MUI)/Multi-Stream Interference (MSI) effects and fast fading effects of multipath propagation tend to disappear; power and bandwidth efficiencies are substantially increased.

This paper deals with SC/FDE for the uplink of a MUMIMO system where a high bandwidth efficiency is intended, through QAM transmission schemes (up to 6 bits/symbol), and the BS is constrained to adopt low-complexity detection techniques, but can be equipped with a large number of receiver antennas, not necessarily much larger than the overall number of transmitter antennas jointly using the same time/frequency resource at mobile terminals. In this context, either a linear detection or a reduced-complexity iterative Decision-Feedback (DF) detection are considered, as presented in sec. II: as to the linear detection alternative, we include the optimum, Minimum Mean-Squared Error (MMSE) detection [7], a reduced-complexity MMSE-type detection [8] and the quite simple Matched Filter (MF) detection; the iterative DF detection alternative, which resorts to joint cancellation of estimated MUI/MSI, is an extension of the iterative DF technique considered by the authors, for 4-QAM transmission, in [9], and can also be regarded as an extension to the multi-input context of reduced-complexity iterative receiver techniques proposed by the authors for SIMO systems (see [10] and the references therein).

In this paper, we evaluate, in detail, the detection performances for the several detection alternatives (discussed with the help of selected performance bounds, as presented in sec. III), for a range of values regarding the number of available BS receiver antennas. A wide set of numerical performance results and the main conclusions of the paper are presented in sections IV and V, respectively. 


\section{MU-Mimo Uplink System Model And Detection TECHNIQUES}

\section{A. SC/FDE with QAM Schemes for Block Transmission}

We consider here a CP-assisted SC/FDE block transmission, within a MU-MIMO system with $N_{T}$ TX antennas and $N_{R}$ RX antennas; for example, but not necessarily, one antenna per MT. We assume, in the $j$ th TX antenna $\left(j=1,2, \ldots, N_{T}\right)$ a length- $N$ block $\mathbf{s}^{(j)}=\left[s_{0}^{(j)}, s_{1}^{(j)}, \ldots, s_{N-1}^{(j)}\right]^{T}$ of time-domain data symbols in accordance with the corresponding binary data block and the selected QAM constellation, concerning $2 m(j)$ bit/symbol, under a Gray mapping rule. A length- $L_{s} \mathrm{CP}$, long enough to cope with the time-dispersive effects of multipath propagation, is also assumed.

By using the frequency-domain version of the time-domain data block $\mathbf{s}^{(j)}$, given by $\mathbf{S}^{(j)}=\left[S_{0}^{(j)}, S_{1}^{(j)}, \cdots, S_{N-1}^{(j)}\right]^{T}=$ $\operatorname{DFT}\left(\mathbf{s}^{(j)}\right)\left(j=1,2, \cdots, N_{T}\right)$, we can describe the frequency-domain transmission rule as follows, for any subchannel $k(k=0,1, \cdots, N-1)$ :

$$
\mathbf{Y}_{k}=\mathbf{H}_{k} \mathbf{S}_{k}+\mathbf{N}_{k}
$$

where $\mathbf{S}_{k}=\left[S_{k}^{(1)}, S_{k}^{(2)}, \cdots, S_{k}^{\left(N_{T}\right)}\right]^{T}$ is the "input vector", $\mathbf{N}_{k}=\left[N_{k}^{(1)}, N_{k}^{(2)}, \cdots, N_{k}^{\left(N_{R}\right)}\right]^{T}$ is the Gaussian noise vector $\left(E\left[N_{k}^{(i)}\right]=0\right.$ and $\left.E\left[\left|N_{k}^{(i)}\right|^{2}\right]=\sigma_{N}^{2}=N_{0} N\right)$, $\mathbf{H}_{k}$ denotes the $N_{R} \times N_{T}$ channel matrix with entries $H_{k}^{(i, j)}$, concerning a given channel realization, and $\mathbf{Y}_{k}=$ $\left[Y_{k}^{(1)}, Y_{k}^{(2)}, \cdots, Y_{k}^{\left(N_{R}\right)}\right]^{T}$ is the resulting, frequency-domain, "output vector".

As to a given MIMO channel realization, it should be noted that the Channel Frequency Response (CFR) $\mathbf{H}^{(i, j)}=\left[H_{0}^{(i, j)}, H_{1}^{(i, j)}, \ldots, H_{N-1}^{(i, j)}\right]^{T}$, concerning the antenna pair $(i, j)$, is the DFT of the Channel Impulse Response $(\mathrm{CIR}) \mathbf{h}^{(i, j)}=\left[h_{0}^{(i, j)}, h_{1}^{(i, j)}, \ldots, h_{N-1}^{(i, j)}\right]^{T}$, where $h_{n}^{(i, j)}=0$ for $n>\operatorname{Ls}(n=0,1, \ldots, N-1)$. Regarding a statistical channel model - which encompasses all possible channel realizations -, let us assume that $E\left[h_{n}^{(i, j)}\right]=0$ and $E\left[h_{n}^{(i, j) *} h_{n^{\prime}}^{(i, j)}\right]=0$ for $n^{\prime} \neq n$. By also assuming, for any $(i, j, k)$, a constant

$$
E\left[\left|H_{k}^{(i, j)}\right|^{2}\right]=\sum_{n=0}^{N-1} E\left[\left|h_{n}^{(i, j)}\right|^{2}\right]=P_{\Sigma}
$$

(of course, with $h_{n}^{(i, j)}=0$ for $n>L_{s}$ ), the average bit energy, regarding the $j t h$ Transmitter (TX) antenna, at each BS antenna, is given by

$$
E_{b}^{(j)}=\frac{\left[\sigma_{s}^{(j)}\right]^{2}}{2 \eta m(j)} P_{\Sigma}
$$

where $\eta=\frac{N}{N+L_{s}}$ and $\left[\sigma_{s}^{(j)}\right]^{2}=E\left[\left|s_{n}^{(j)}\right|^{2}\right]$.

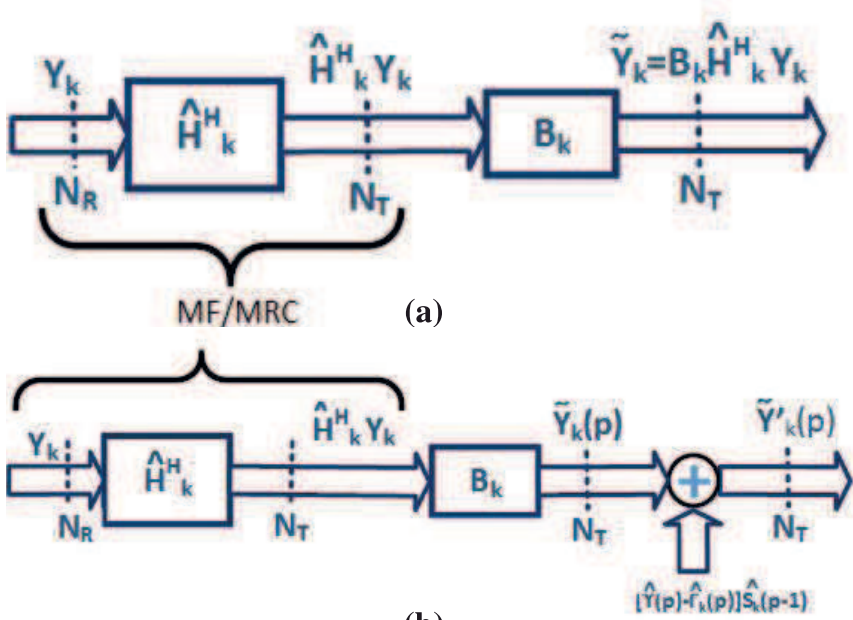

(b)

Fig. 1. Frequency-domain linear detection (a) and iterative DF detection combining a linear detection and interference cancellation in the frequency domain (b).

\section{B. Linear Detection Techniques}

An appropriate linear detector can be implemented by resorting to frequency-domain processing. After $\mathrm{CP}$ removal, a DFT operation leads to the required set $\left\{\mathbf{Y}_{k} ; k=0,1, \cdots, N-1\right\}$ of length- $N_{R}$ inputs to the frequency-domain detector ( $\mathbf{Y}_{k}$ given by (1)); it works, for each $k$, as shown in Fig. 1(a), leading to a set $\left\{\tilde{\mathbf{Y}}_{k} ; k=0,1, \cdots, N-1\right\}$ of length- $N_{T}$ outputs $\tilde{\mathbf{Y}}_{k}=$ $\left[\tilde{Y}_{k}^{(1)}, \tilde{Y}_{k}^{(2)}, \cdots, \tilde{Y}_{k}^{\left(N_{T}\right)}\right]^{T} \quad(k=0,1, \cdots, N-1)$.

When $N_{T} \leq N_{R}$, possibly with $N_{R} \gg 1$, either an MMSE, frequency-domain, optimum linear detection or a reduced-complexity, frequency-domain, linear detection can be considered. In all cases, the detection matrix, for each subchannel $k(k=0,1, \ldots, N-1)$ can be written as

$$
\mathbf{D}_{k}=\mathbf{B}_{k} \widehat{\mathbf{H}}_{k}^{H},
$$

where $\widehat{\mathbf{H}}_{k}^{H}$ is the conjugate transpose of the estimated MUMIMO channel matrix $\widehat{\mathbf{H}}_{k}$ and $\mathbf{B}_{k}$ is a selected $N_{T} \times N_{T}$ matrix, possibly depending on $\widehat{\mathbf{H}}_{k}$. Therefore, $\widetilde{\mathbf{Y}}_{k}=\mathbf{D}_{k} \mathbf{Y}_{k}=$ $\mathbf{B}_{k} \widehat{\mathbf{H}}_{k}^{H} \mathbf{Y}_{k}$ at the output of the frequency-domain linear detector (see Fig. 1(a)).

It should be noted that the $j$ th component of $\widehat{\mathbf{H}}_{k}^{H} \mathbf{Y}_{k}$ is given by $\sum_{i=1}^{N_{R}} \widehat{H}_{k}^{(i, j) *} Y_{k}^{(i)}\left(j=1,2, \cdots, N_{T}\right)$ : this means that the $\widehat{\mathbf{H}}_{k}^{H}$ factor provides $N_{T}$ Maximal Ratio Combining (MRC) procedures, one per MT antenna, all of them based on an appropriate MF for each component of the length- $N_{R}$ received vector at subchannel $k$.

For an MMSE detection -the optimum linear detection-, we need to adopt $\mathbf{B}_{k}=\mathbf{A}_{k}^{-1}$, where

$$
\mathbf{A}_{k}=\widehat{\mathbf{H}}_{k}^{H} \widehat{\mathbf{H}}_{k}+\widehat{\boldsymbol{\alpha}}
$$

with $\hat{\boldsymbol{\alpha}}$ denoting a diagonal matrix characterized by the estimated values of $\alpha^{(j)}=\frac{N_{o}}{\left[\sigma_{s}^{(j)}\right]^{2}}\left(j=1,2, \ldots N_{T}\right)$. By 
simply adopting $\mathbf{B}_{k}=\mathbf{I}_{N_{T}}$ (an $N_{T} \times N_{T}$ identity matrix), we can strongly reduce the implementation complexity: since $\mathbf{D}_{k}=\mathbf{B}_{k} \widehat{\mathbf{H}}_{k}^{H}=\widehat{\mathbf{H}}_{k}$, this is a MF detection.

When replacing the MMSE detection by a simple MF detection, however, a significant performance degradation is unavoidable. An intermediate detection solution -regarding both complexity and performance - is provided by the adoption of a simplified MMSE-type scheme which replaces the exact inversion of $\mathbf{A}_{k}$ by an approximate inversion. In this context, we consider a recommendation of [8] so as to obtain $\mathbf{B}_{k} \approx$ $\mathbf{A}_{k}^{-1}$ while avoiding a complex $N_{T} \times N_{T}$ matrix inversion: $\mathbf{B}_{k}=\left(\operatorname{diag}\left(\mathbf{A}_{k}\right)\right)^{-1} \times$

$$
\left[\mathbf{I}_{N_{T}}-\left(\mathbf{A}_{k}-\operatorname{diag}\left(\mathbf{A}_{k}\right)\right)\left(\operatorname{diag}\left(\mathbf{A}_{k}\right)\right)^{-1}\right],
$$

where $\mathbf{A}_{k}$ is given by (5) and $\operatorname{diag}\left(\mathbf{A}_{k}\right)$ is a diagonal $N_{T} \times N_{T}$ matrix (very easy to invert) which shares the main diagonal with $\mathbf{A}_{k}$. By defining $\mathbf{G}_{k}=\mathbf{H}_{k}^{H} \mathbf{H}_{k}$-and $\widehat{\mathbf{G}}_{k}=\widehat{\mathbf{H}}_{k}^{H} \widehat{\mathbf{H}}_{k^{-}}, \mathbf{B}_{k}$ can be written as a function of $\widehat{\mathbf{G}}_{k}$ and $\widehat{\boldsymbol{\alpha}}$ with both the standard MMSE detection and the simplified MMSE-type detection reported above, since $\mathbf{A}_{k}=\widehat{\mathbf{G}}_{k}+\widehat{\boldsymbol{\alpha}}$.

For a given channel realization $\mathbf{H}_{k}$ and a given detection matrix $\mathbf{D}_{k}$, which depends on the estimated channel realization $\widehat{\mathbf{H}}_{k}$, the output of the frequency-domain detector is given by

$$
\tilde{\mathbf{Y}}_{k}=\mathbf{D}_{k} \mathbf{Y}_{k}=\boldsymbol{\Gamma}_{k} \mathbf{S}_{k}+\mathbf{N}_{k}^{\prime},
$$

where $\boldsymbol{\Gamma}_{k}=\mathbf{D}_{k} \mathbf{H}_{k}$ and $\mathbf{N}_{k}^{\prime}=\mathbf{D}_{k} \mathbf{N}_{k}$.

With SC/FDE (time-domain data symbols), an Inverse Discrete Fourier Transform (IDFT) is required for each $\tilde{\mathbf{Y}}^{(j)}=\left[\widetilde{Y}_{0}^{(j)}, \tilde{Y}_{1}^{(j)}, \cdots, \tilde{Y}_{N-1}^{(j)}\right]^{T}$ vector. The $n$th component of the resulting length- $N \operatorname{IDFT}\left(\widetilde{\mathbf{Y}}^{(j)}\right)=\widetilde{\mathbf{y}}^{(j)}$ vector can be written as $\widetilde{y}_{n}^{(j)}=\gamma^{(j)} s_{n}^{(j)}+I S I+$ $M U I / M S I+{ }^{\prime}$ Gaussian noise', with $\gamma^{(j)}=\frac{1}{N} \sum_{k=0}^{N-1} \Gamma_{k}^{(j, j)}$ $\left(\gamma^{(j)}=E\left[\widetilde{y}_{n}^{(j)} s_{n}^{(j) *}\right] /\left[\sigma_{s}^{(j)}\right]^{2}\right)$. Therefore we can write

$$
\tilde{\mathbf{Y}}_{k}=\gamma \mathbf{S}_{k}+\left(\boldsymbol{\Gamma}_{k}-\gamma\right) \mathbf{S}_{k}+\mathbf{D}_{k} \mathbf{N}_{k}
$$

where $\gamma$ is a diagonal $N_{T} \times N_{T}$ matrix with $(j, j)$ entries given by $\gamma^{(j)}=\frac{1}{N} \sum_{k=0}^{N-1} \Gamma_{k}^{(j, j)}$. With $\widetilde{Y}_{k}^{(j)}$ written as

$$
\begin{aligned}
\tilde{Y}_{k}^{(j)}= & \gamma^{(j)} S_{k}^{(j)}+\left[\Gamma_{k}^{(j, j)}-\gamma^{(j)}\right] S_{k}^{(j)}+ \\
& \sum_{\substack{l=1 \\
(l \neq j)}}^{N_{T}} \Gamma_{k}^{(j, l)} S_{k}^{(l)}+\sum_{i=1}^{N_{R}} D_{k}^{(j, i)} N_{k}^{(i)},
\end{aligned}
$$

the terms in the right-hand side of eq. (9) are concerned, respectively, to "signal", Inter-Symbol Interference (ISI), MUI/MSI and "Gaussian noise", at subchannel $k$.

\section{Low-Complexity Iterative DF Detection Technique}

A low-complexity iterative DF technique can be easily devised having in mind eq. (8). This frequency-domain nonlinear detection technique is depicted in Fig. 1(b). It combines the use of a linear detector and, for all iterations after the initial iteration (i.e., for $p>1$ ), a cancellation of residual MUI - and residual MSI, when some users adopt several TX antennas for spatial multiplexing purposes - as well as residual ISI; such cancellation is based on the estimated data block which is provided by the preceding iteration and fed back to the frequency-domain detector. The output of this detector, for iteration $p$, is

$$
\widetilde{\mathbf{Y}}_{k}^{\prime}(p)=\widetilde{\mathbf{Y}}_{k}(p)+\left[\widehat{\gamma}(p)-\widehat{\boldsymbol{\Gamma}}_{k}(p)\right] \widehat{\mathbf{S}}_{k}(p-1),
$$

$\left[k=0, \cdots, N-1 ; p>1\left(\right.\right.$ for $\left.\left.p=1, \tilde{\mathbf{Y}}_{k}^{\prime}(p)=\widetilde{\mathbf{Y}}_{k}(p)\right)\right]$,

where $\widehat{\boldsymbol{\Gamma}}_{k}(p)=\mathbf{D}_{k}(p) \widehat{\mathbf{H}}_{k}$ - with $\mathbf{D}_{k}(p)$ denoting the detection matrix employed in iteration $p$ - and the entries $(j, j)$ of the diagonal matrix $\widehat{\gamma}(p)$ are given by $\widehat{\gamma}^{(j)}(p)=\frac{1}{N} \sum_{k=0}^{N-1} \widehat{\Gamma}_{k}^{(j, j)}(p)$. Of course, $\quad\left[\widehat{S}_{0}^{(j)}(p-1), \cdots, \widehat{S}_{N-1}^{(j)}(p-1)\right]^{T}=$ $\operatorname{DFT}\left(\left[\widehat{s}_{0}^{(j)}(p-1), \cdots, \widehat{s}_{N-1}^{(j)}(p-1)\right]^{T}\right)$.

The implementation of this iterative DF technique is especially simple when $\mathbf{D}_{k}(p)=\widehat{\mathbf{H}}_{k}^{H}$ for any $p$, i.e., with a linear MF detector for all iterations; an interesting alternative, which also avoids a complex matrix inversion, consists of adopting $\mathbf{B}_{k}(p)=\mathbf{I}_{N_{T}}$ for $p>1$ (i.e., MF detection) and, for $p=1, \mathbf{B}_{k}(p)$ as given by eq. (6). It should be noted that $\widehat{\boldsymbol{\Gamma}}_{k}(p)=\mathbf{D}_{k}(p) \widehat{\mathbf{H}}_{k}=\mathbf{B}_{k}(p) \widehat{\mathbf{H}}_{k}^{H} \widehat{\mathbf{H}}_{k}=\mathbf{B}_{k}(p) \widehat{\mathbf{G}}_{k}$ : $\widehat{\boldsymbol{\Gamma}}_{k}(p)=\widehat{\mathbf{G}}_{k}$ for the MF detector and $\widehat{\boldsymbol{\Gamma}}_{k}(p)$ is a function of $\widehat{\mathbf{G}}_{k}$ and $\widehat{\boldsymbol{\alpha}}$ for the simplified MMSE-type detector.

\section{On Achievable Detection Performances When Many BS Antennas are AVailable}

\section{A. Bit Error Rate (BER) with Linear Detection}

Regarding evaluation of linear detection performances by simulation, a simple semi-analytical method is presented here, combines simulated channel realizations and analytical computations of BER performance which are conditional on those channel realizations. In all cases, the conditional BER values are directly computed by resorting to a Signal-to-Interferenceplus-Noise-Ratio (SINR), under the realistic assumption that the "interference" has a quasi-Gaussian nature. These ratios are simply derived in accordance with the channel realization $\mathbf{H}_{k}(k=0,1, \cdots, N-1)$. Of course, for concluding the BER computation in each case - involving random generation of a large number of channel realizations and conditional BER computations - a complementary averaging operation over the set of channel realizations is required.

When using a linear detection technique (sec. II-B), it is easy to conclude, having in mind [10], that the "signal-tointerference-plus-noise" ratio concerning the $j$ th input of the MU-MIMO system is given by

$$
S I N R_{j}=\frac{N\left|\gamma^{(j)}\right|^{2}}{\beta_{j}+\sum_{\substack{l=1 \\(l \neq j)}}^{N_{T}} \beta_{l}+\alpha^{(j)} \sum_{i=1}^{N_{R}} \sum_{k=0}^{N-1}\left|D_{k}^{(j, i)}\right|^{2}}
$$


where $\alpha^{(j)}=\frac{N_{0}}{\left[\sigma_{s}^{(j)}\right]^{2}}=\frac{P_{\Sigma}}{2 \eta m(j) \frac{E_{b}^{(j)}}{N_{0}}}, \quad \beta_{j}$
$\sum_{k=0}^{N-1}\left|\Gamma_{k}^{(j, j)}-\gamma^{(j)}\right|^{2}, \beta_{l}=\frac{\left[\sigma_{s}^{(l)}\right]^{2}}{\left[\sigma_{s}^{(j)}\right]^{2}} \sum_{k=0}^{N-1}\left|\Gamma_{k}^{(j, l)}\right|^{2}(l \neq j)$.

For 4-QAM transmission $(m(j)=1)$, the resulting $B E R_{j}$ $\left(j=1,2, \cdots, N_{T}\right)$ - conditional on the channel realization $\left\{\mathbf{H}_{k} ; k=0,1, \cdots, N-1\right\}$ - is then given by

$$
B E R_{j} \approx Q\left(\sqrt{S I N R_{j}}\right)
$$

where $Q($.$) is the Gaussian error function.$

For 16-QAM transmission $(m(j)=2)$, the resulting $B E R_{j}$ $\left(j=1,2, \cdots, N_{T}\right)$ - can be obtained very accurately as follows (having in mind the Gray mapping rule):

$$
B E R_{j}=\frac{1}{2}\left[B E R_{j, 1}+B E R_{j, 2}\right]
$$

where $B E R_{j, 1}=\frac{1}{2} p_{1}+\frac{1}{2} p_{3}$ and $B E R_{j, 2}=\frac{1}{2}\left(p_{1}+p_{3}\right)+$ $\frac{1}{2}\left(p_{1}-p_{5}\right)$ with

$$
p_{n} \approx Q\left(\sqrt{\frac{n^{2}}{5} S I N R_{j}}\right) .
$$

For 64-QAM transmission $(m(j)=3)$, an accurate approximation to $B E R_{j}$ can also be obtained (also having in mind the Gray mapping rule):

$$
B E R_{j}=\frac{1}{3}\left[B E R_{j, 1}+B E R_{j, 2}+B E R_{j, 3}\right]
$$

where $B E R_{j, 1}=\frac{1}{4} p_{1}^{\prime}+\frac{1}{4} p_{3}^{\prime}+\frac{1}{4} p_{5}^{\prime}+\frac{1}{4} p_{7}^{\prime}, B E R_{j, 2}=$ $\frac{1}{4}\left(p_{3}^{\prime}-p_{11}^{\prime}\right)+\frac{1}{4}\left(p_{1}^{\prime}-p_{9}^{\prime}\right)+\frac{1}{4}\left(p_{1}^{\prime}+p_{7}^{\prime}\right)+\frac{1}{4}\left(p_{3}^{\prime}+p_{5}^{\prime}\right)$ and $B E R_{j, 3}=\frac{1}{4}\left(p_{1}^{\prime}-p_{5}^{\prime}+p_{9}^{\prime}-p_{13}^{\prime}\right)+\frac{1}{4}\left(p_{1}^{\prime}+p_{3}^{\prime}-p_{7}^{\prime}+p_{11}^{\prime}\right)+$ $\frac{1}{4}\left(p_{3}^{\prime}+p_{1}^{\prime}-p_{5}^{\prime}+p_{9}^{\prime}\right)+\frac{1}{4}\left(p_{1}^{\prime}-p_{5}^{\prime}+p_{3}^{\prime}-p_{7}^{\prime}\right)$, with

$$
p_{n}^{\prime} \approx Q\left(\sqrt{\frac{n^{2}}{21} S I N R_{j}}\right) .
$$

When $n>1$, of course, $p_{n} \ll p_{1}$ and $p_{n}^{\prime} \ll p_{1}^{\prime}$ for high $S I N R_{j}$. By using $M(j)=2^{m(j)}$, we get

$$
B E R_{j} \approx \frac{2}{m(j)}\left(1-\frac{1}{M(j)}\right) Q\left(\sqrt{3 \frac{S I N R_{j}}{[M(j)]^{2}-1}}\right)
$$

\section{B. IBER Levels under Low-Complexity Constraints}

When a low-complexity linear detection technique is adopted - such as the MF detection or the simplified MMSEtype detection reported in subsection II-B -, an "error floor" effect is unavoidable. The corresponding Irreducible BER is given by $I B E R_{j}=\lim _{\alpha^{(j)} \rightarrow 0} B E R_{j}=$

$$
\lim _{\alpha^{(j)} \rightarrow 0} f\left(S I N R_{j}\right)=f\left(\frac{N\left|\gamma^{(j)}\right|^{2}}{\beta_{j}+\sum_{l=1(l \neq j)}^{N_{T}} \beta_{l}}\right)
$$

where $f\left(S I N R_{j}\right)$ is the appropriate function of $S I N R_{j}$, according to $m(j)$, which is used for computation of $B E R_{j}$, as explained in Sub-Section III-A $\left(\gamma^{(j)}, \beta_{j}\right.$ and $\beta_{l}(l \neq j)$ as in $\left.(11)\right)$.

\section{SIMO Performances Bounds and Massive MIMO effects}

The $S I N R_{j}$ for semi-analytical computation of the SIMO/MFB (SIMO/MFB) on detection performance can be easily derived from (11), by considering the Gaussian noise and the "useful" term, at the MF detector output, but not the two interference terms (MUI/MSI, ISI). Therefore, the SIMO/MFB bound is given by the appropriate $f\left(S I N R_{j}\right)$, according to $m(j)$, with $S I N R_{j}=$

$$
\frac{N\left|\gamma^{(j)}\right|^{2}}{\alpha^{(j)} \sum_{k=0}^{N-1} \sum_{i=1}^{N_{R}}\left|H_{k}^{(i, j)}\right|^{2}}=\frac{1}{N \alpha^{(j)}} \sum_{k=0}^{N-1} \sum_{i=1}^{N_{R}}\left|H_{k}^{(i, j)}\right|^{2}
$$

since $\gamma^{(j)}=\frac{1}{N} \sum_{k=0}^{N-1} \sum_{i=1}^{N_{R}}\left|H_{k}^{(i, j)}\right|^{2}$ with MF detection.

By assuming a LOS single-path radio propagation for each $(i, j)$ antenna pair -i.e., $\left|H_{k}^{(i, j)}\right|^{2}=P_{\Sigma}$-, we can obtain

$$
S I N R_{j}=\frac{1}{\alpha^{(j)}} N_{R} P_{\Sigma}=2 \eta m(j) N_{R} \frac{E_{b}^{(j)}}{N_{0}}
$$

leading to the SIMO/AWGN/MFB.

When $N_{R} \gg N_{T}$, both the MUI/MSI effects and the effects of multipath propagation (fading, ISI) tend to disappear: consequently, the BER performances for the MUMIMO $N_{T} \times N_{R}$ Rayleigh fading channel become very close to those concerning a SIMO $1 \times N_{R}$ channel with single-path propagation for all $N_{R} \mathrm{TX} / \mathrm{RX}$ antenna pairs. The "massive MIMO" effects that can be explained, when $N_{R} \gg N_{T}$, mean that $\sum_{i=1}^{N_{R}}\left|H_{k}^{(i, j)}\right|^{2} \approx N_{R} P_{\Sigma}$ and $\sum^{N_{T}} H_{k}^{(i, j) *} H_{k}^{(i, l)} \approx 0$, leading to $B E R_{j}=$ $l=1(l \neq j)$

$f\left(S I N R_{j}\right)$ with $S I N R_{j} \approx 2 \eta m(j) N_{R} \frac{E_{b}^{(j)}}{N_{0}}$ (i.e., closely approximating the SIMO/AWGN/MFB).

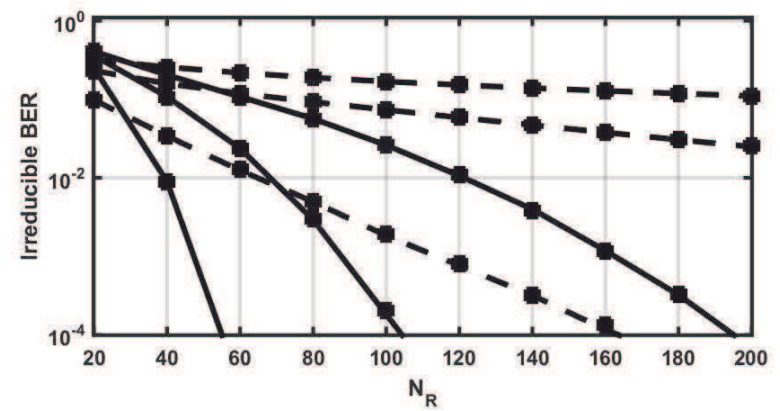

Fig. 2. IBER performance curves concerning either MF detection (dashed lines) or simplified MMSE-type detection (solid lines), for 4-QAM (the best), 16-QAM and 64-QAM (the worst).

\section{Numerical RESUlts AND Discussion}

The set of performance results which are presented here are concerned to SC/FDE uplink block transmission using QAM (up to $6 \mathrm{bit} / \mathrm{symbol}$ ), with $N=256$ and $L s=64$ in a MU-MIMO $N_{T} \times N_{R}$ Rayleigh fading channel with 


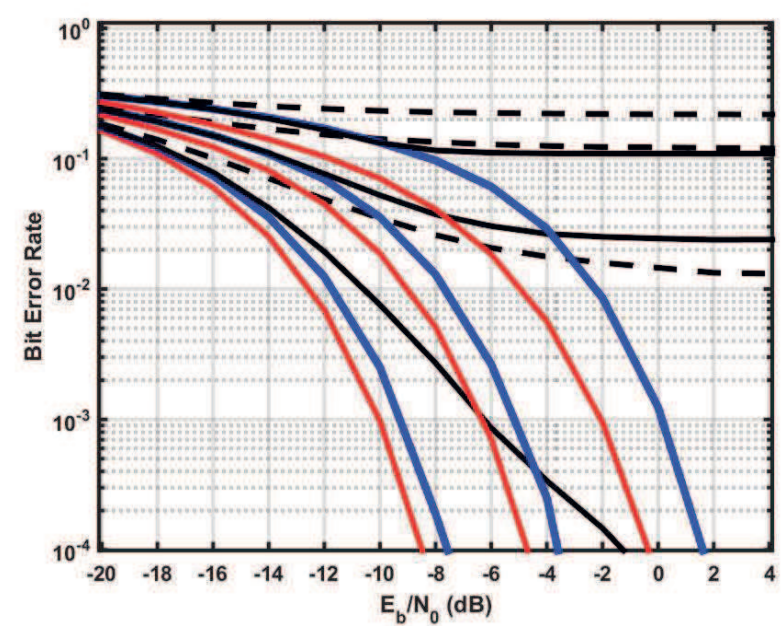

(a)

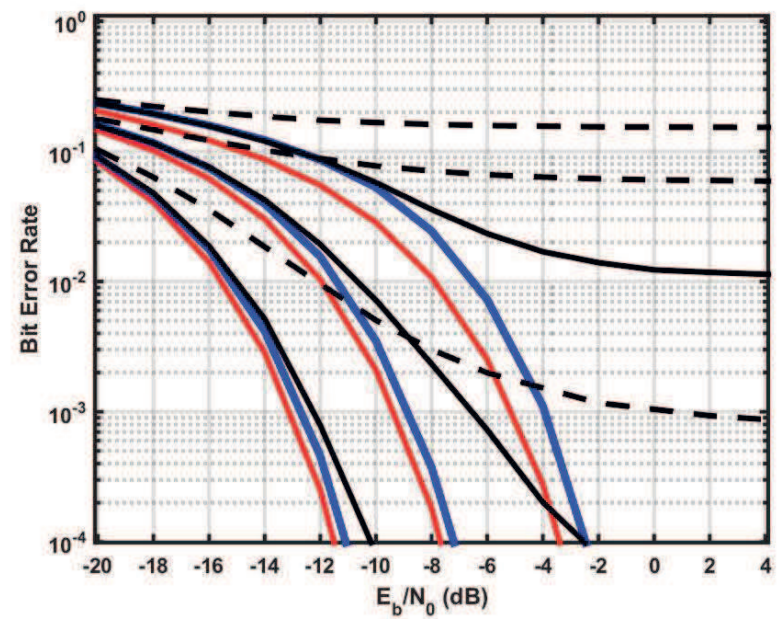

(b)

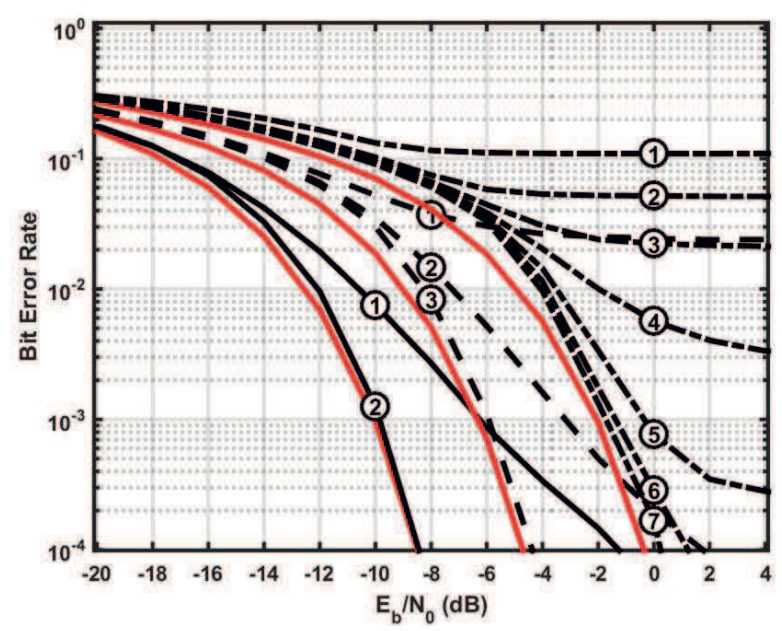

(a)

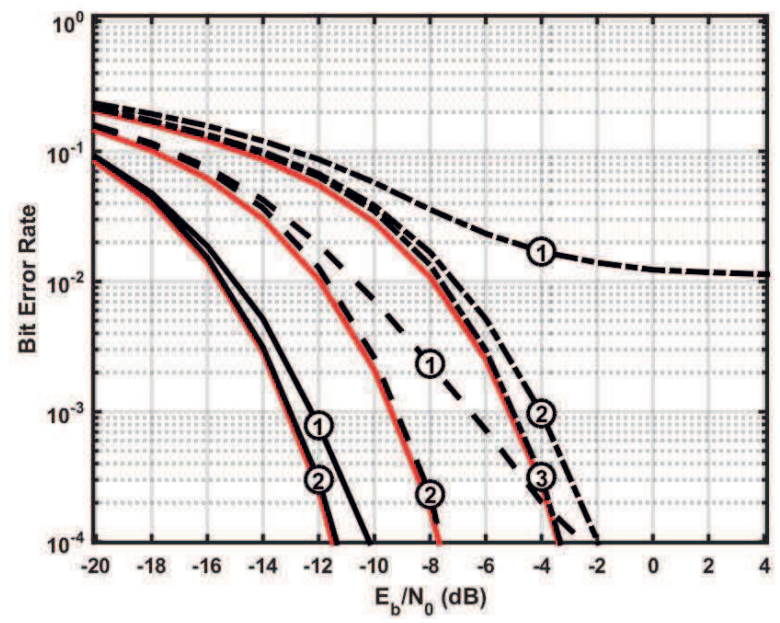

(b)

Fig. 3. BER performances - when $N_{R}=60$ (a) or 120 (b) - concerning MF Fig. 4. BER performances - when $N_{R}=60$ (a) or 120 (b) - concerning an detection (black dashed lines), simplified MMSE-type detection (black solid iterative DF detection, with a simplified MMSE-type detector for $p=1$ and an lines) or true MMSE detection (blue lines), for 4-QAM (the best), 16-QAM and MF detector for $p>1$, for 4-QAM (black solid lines), 16-QAM (dashed lines) 64-QAM (the worst) [SIMO/AWGN/MFB reference performances (red lines) and 64-QAM (dot-dashed lines) [SIMO/AWGN/MFB reference perpormances are also included.] (red lines) are also included.]

$N_{T}=12$. Perfect channel estimation and power control are assumed. The fading effects regarding the several TX/RX antenna pairs are supposed to be uncorrelated, with independent zero-mean complex Gaussian $h_{n}^{(i, j)}$ coefficients assumed to have variances $P_{n}=1-n / 63, n=0, \ldots, 63\left(P_{n}=0\right.$ for $n=64, \ldots, 255)$.

Fig. 2 shows Irreducible BER (IBER) results for a wide range of values of $N_{R}$ and the same QAM constellation (4, 16 or $64-\mathrm{QAM}$ ) at all $N_{T}=12$ inputs of the MU-MIMO system. These performance curves have been obtained through the semi-analytical simulation approach presented in sec. III, and confirmed by conventional Monte-Carlo simulation (quasisuperposed dots), involving an error-counting procedure. Two alternative low-complexity linear detection techniques were considered, both avoiding complex $12 \times 12$ matrix inversions: the MF detection and the simplified MMSE-type detection, as reported in sec. II. These results clearly show that, under both low-complexity detection techniques, unacceptably high error floor levels (say, IBER $>10^{-1}$ ) are unavoidable if $N_{R}$ is not large enough; they become especially high when a high bandwidth efficiency is intended - through an increased size of the QAM constellation - and/or the MF detection is adopted. An excellent agreement of performance results obtained by semi-analytical means and by conventional Monte Carlo simulations is also observed.

Fig. 3 shows BER performances - obtained through the semi-analytical method - with $N_{R}=60$ (a) or 120 (b), the same QAM constellation and the same $\sigma_{s}^{(j)}$ at all $N_{T}=12$ system inputs, and a linear detection technique: MF detection, simplified MMSE-type detection or true MMSE detection 


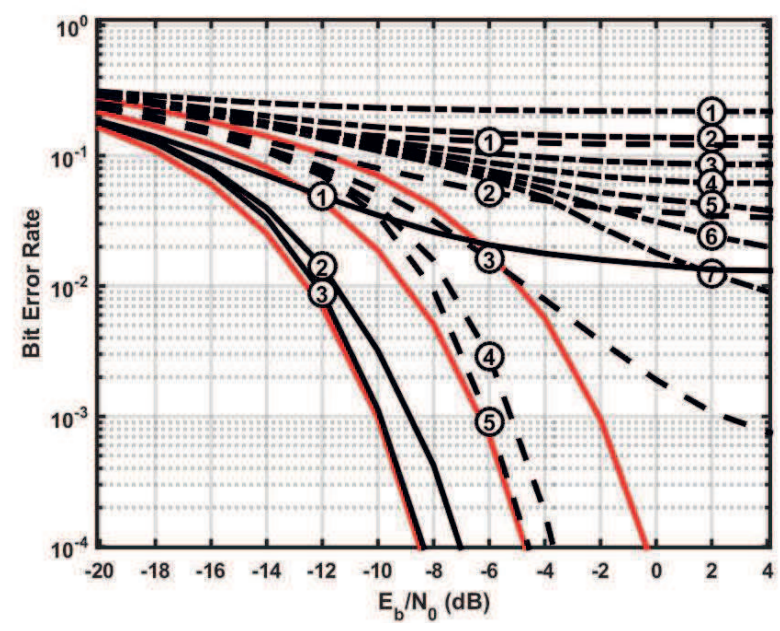

(a)

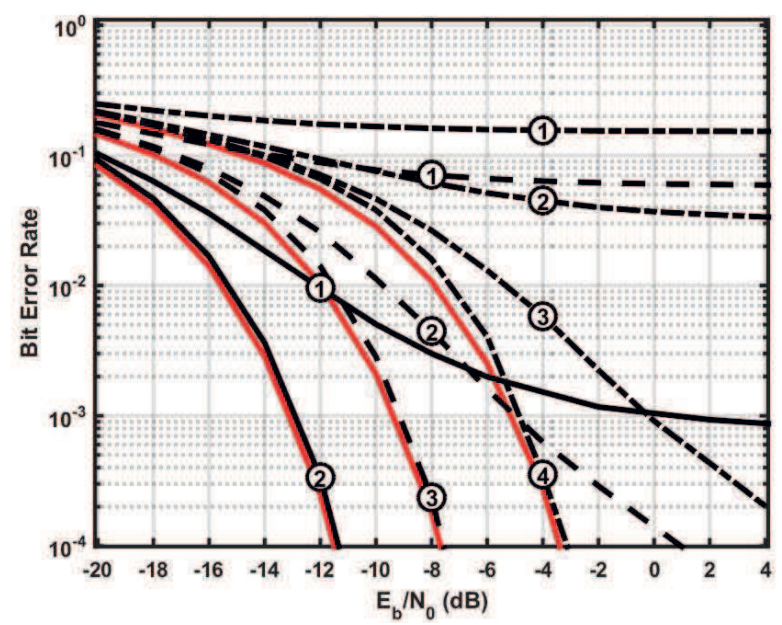

(b)

Fig. 5. Similar to Fig. 4, but assuming an MF detector for all iterations.

(see subsection II-B). For comparisons, we also include the analytical SIMO/AWGN/MFB. These results confirm those of Fig. 2 on the performance limitations of the low-complexity linear detection techniques (designed to avoid a complex $12 \times 12$ matrix inversion), also showing the good performances achievable through a linear ideal MMSE detection (which requires a complex $12 \times 12$ matrix inversion), with a nice approximation to the SIMO/AWGN/MFB (a gap below $2 \mathrm{~dB}$, even for $N_{R}=60$ and $64-\mathrm{QAM}$ ).

Fig. 4 shows the simulated BER performances - obtained through the conventional Monte Carlo method - with $N_{R}=60$ (a) or 120 (b), the same QAM constellation used at all $N_{T}=$ 12 system inputs, and an iterative DF detection technique as described in subsection II-C . A simplified MMSE-type detector is adopted for the first iteration (i.e. $\mathbf{B}_{k}(p)$ is given by eq. (6) for $p=1$ ) and an MF detector is adopted for the remaining iterations (i.e. $\mathbf{B}_{k}(p)=\mathbf{I}_{N_{T}}$ for $p>1$ ). Similarly to Fig. 3, this figure also includes analytical SIMO/AWGN/MFB results. In the closely related Fig. 5, also concerning an iterative DF detection as described in subsection II-C, we adopted an MF detector for all iterations.

Figures 4 and 5 show that, by combining the use of the low-complexity linear detectors with an interference cancellation procedure, as depicted in Fig. 1(b), a close approximation to the SIMO/MFB (and the practically identical SIMO/AWGN/MFB) can be achieved: after a few iterations, the performance gap, at $B E R=10^{-3}$, becomes negligible, if required by using the simplified MMSE-type detection instead of the MF detection - in the first iteration.

\section{CONCLUSIONS}

This paper was dedicated to uplink detection issues concerning a MU-MIMO system where QAM-based (up to 6 bits/symbol) SC/FDE transmission schemes are adopted and a large number of BS antennas is available, possibly much larger than the corresponding number of transmitter antennas at mobile terminals. In this context, we considered several detection techniques and evaluated, in detail, the resulting performances (discussed with the help of selected performance bounds), for a range of values regarding the number of BS antennas.

From our performance results, our main conclusion is that simple linear detection techniques, designed to avoid the need of complex matrix inversions, can lead to unacceptably high error floor levels; however, by combining the use of such simple linear detectors with an interference cancellation procedure - within a low-complexity iterative DF technique -, a close approximation to the SIMO MFB performance can be achieved, even for 64-QAM schemes, after a few iterations.

\section{REFERENCES}

[1] A. Gusmao, R. Dinis, J. Conceicao, and N. Esteves. Comparison of Two Modulation Choices for Broadband Wireless Communications. In VTC 2000-Spring. IEEE 51st, volume 2, pages 1300-1305, May 2000.

[2] G. Foschini. Layered Space-Time Architecture for Wireless Communication in a Fading Environment when Using Multi-element Antennas. Bell Labs Technology Journal, vol. 1 (2), 1996.

[3] Gesbert et al. Shifting the MIMO Paradigm. Signal Processing Magazine, IEEE, 24(5):36-46, Sept 2007.

[4] T.L. Marzetta. Noncooperative Cellular Wireless with Unlimited Numbers of Base Station Antennas. Wireless Communications, IEEE Transactions on, 9(11):3590-3600, November 2010.

[5] Rusek et al. Scaling Up MIMO: Opportunities and Challenges with Very Large Arrays. Sign. Proc. Mag., IEEE, 30(1):40-60, Jan 2013.

[6] J. Hoydis, S. ten Brink, and M. Debbah. Massive MIMO in the UL/DL of Cellular Networks: How Many Antennas Do We Need? Sel. Areas in Comm., IEEE Journal on, 31(2):160-171, Feb 2013.

[7] N. Kim, Y. Lee, and H. Park. Performance Analysis of MIMO System with Linear MMSE Receiver. Wireless Comm., IEEE Trans. on, 7(11):4474-4478, Nov 2008.

[8] $\mathrm{Wu}$ et al. Approximate matrix inversion for high-throughput data detection in the large-scale MIMO uplink. In Circuits and Systems (ISCAS), 2013 IEEE Int. Symp. on, pages 2155-2158, May 2013.

[9] P. Torres, L. Charrua, and A. Gusmao. On Detection Issues in the SCbased Uplink of a MU-MIMO System with a Large Number of BS Antennas. IEEE Vehicular Technology Conf., VTC, Sep 2015.

[10] A Gusmao, P. Torres, R. Dinis, and N. Esteves. A Turbo FDE Technique for Reduced-CP SC-Based Block Transmission Systems. Comm., IEEE Trans. on, 55(1):16-20, Jan 2007. 